\title{
Weak evidence supports the short-term benefits of orthopaedic treatment for Class III malocclusion in children
}

\section{Abstracted from \\ Watkinson S, Harrison JE, Furness S, Worthington HV.}

Orthodontic treatment for prominent lower front teeth (Class III malocclusion) in children. Cochrane Database Syst Rev 2013; 9: Art. No.: CD003451. DOI: 10.1002/14651858.CD003451.pub2.

Address for correspondence: Luisa Fernandez Mauleffinch, Review Group Co-ordinator, Cochrane Oral Health Group, MANDEC, School of Dentistry, University of Manchester, Higher Cambridge Street, Manchester, M15 6FH, UK. E-mail: luisa.fernandez@manchester.ac.uk

\section{Question: What is the most effective treatment for class III malocclusion in children?}

Data sources The Cochrane Oral Health Group's Trials Register, the Cochrane Central Register of Controlled Trials (CENTRAL), Medline and Embase.

Study selection Randomised controlled trials (RCTs) of orthodontic treatment to correct prominent lower front teeth were included.

Data extraction and synthesis Study screening, risk of bias assessment and data extraction were carried out independently by two reviewers. The mean differences with $95 \%$ confidence intervals were calculated for continuous data. Meta-analysis was undertaken when studies of similar comparisons reported comparable outcome measures. A fixed-effect model was used. The $I^{2}$ statistic was used as a measure of statistical heterogeneity.

Results Seven RCTs (339 patients) were included in this review. One study was assessed as at low risk of bias, three at high risk of bias and three at unclear risk. Four studies reported on the use of a facemask, two on the chin cup, one on the tandem traction bow appliance and one on mandibular headgear.

One study reported on both the chin cup and mandibular headgear appliances. One study ( $n=73$, low quality evidence), comparing a facemask to no treatment, reported a mean difference (MD) in overjet of $4.10 \mathrm{~mm}$ (95\% confidence interval $(\mathrm{Cl}) 3.04$ to 5.16; $\mathrm{P}$ value $<$ $0.0001)$ favouring the facemask treatment.

Three studies ( $n=155$, low quality evidence) reported ANB differences immediately after treatment with a facemask when compared to an untreated control. The pooled data showed a statistically significant MD in ANB in favour of the facemask of $3.93^{\circ}$ (95\% Cl 3.46 to $4.39 ; \mathrm{P}$ value $<0.0001$ ). There was significant

This paper is based on a Cochrane Review published in the Cochrane Library 2013, issue 9 (see www.thecochranelibrary.com for information). Cochrane Reviews are regularly updated as new evidence emerges and in response to feedback, and the Cochrane Library should be consulted for the most recent version of the review. heterogeneity between these studies $(12=82 \%)$. This is likely to have been caused by the different populations studied and the different ages at the time of treatment.

One study ( $n=73$, low quality evidence) reported outcomes of the use of the facemask compared to an untreated control at three years follow-up. This study showed that improvements in overjet and ANB were still present three years post-treatment. In this study, adverse effects were reported, but due to the low prevalence of temporomandibular (TMJ) signs and symptoms no analysis was undertaken.

Two studies ( $n=90$, low quality evidence) compared the chin cup with an untreated control. Both studies found a statistically significant improvement in ANB, and one study also found an improvement in the Wits appraisal. Data from these two studies were not suitable for pooling.

A single study of the tandem traction bow appliance compared to untreated control ( $n=30$, very low quality evidence) showed a statistically significant difference in both overjet and ANB favouring the intervention group. The remaining two studies did not report the primary outcome of this review.

Conclusions There is some evidence that the use of a facemask to correct prominent lower front teeth in children is effective when compared to no treatment on a short-term basis. However, in view of the general poor quality of the included studies, these results should be viewed with caution. Further randomised controlled trials with long follow-up are required.

\section{Commentary}

Class III malocclusion, originating from dental and/or skeletal factors, often exhibits reverse overjet and/or sagittal skeletal discrepancy. It involves diverse treatment modalities ranging from an orthopaedic approach to orthodontic camouflage and definitive orthognathic treatment. Treatment approaches could not be initiated until the exact aetiologies (dental, skeletal or both) are clarified. This systematic review suggests that orthopaedic treatments are beneficial for class III malocclusion in children in the short term. 
Seven eligible studies were included but were found to report multiple interventions and various outcomes, which made metaanalysis possible for only one outcome in the comparison between facemask and no treatment. Despite this, the authors followed a sound methodological approach to critically appraise the evidence from included studies. The authors evaluated the risk of bias for each included study and the quality of evidence for each outcome assessed, which resulted in an assessment of an overall low quality of evidence in this systematic review.

This systematic review aimed to include all possible orthodontic treatments for class III malocclusion, but finally only orthopaedic approaches were included. As mentioned above, appropriate treatment modality could not be initiated until exact aetiologies were determined. Orthopaedic appliances for class III malocclusion should be indicated for children with skeletal discrepancy, while orthodontic approaches (for example, fixed appliances) would suffice for those with only dental problems. However, the inclusion criteria of skeletal discrepancies were not fully implemented among the included studies (for example, Vaughn ${ }^{1}$ ) in this systematic review, which may disguise the true effects of orthopaedic treatments for children with skeletal discrepancy, and further downgrades the quality of evidence in this systematic review.

Providing the possibility of relapse, it is thoughtful of the authors to stratify the treatment effects into short-term and long-term.
This review aims to determine whether orthodontic treatments in children would reduce the need for orthognathic surgery after skeletal maturation. Nevertheless, the longest follow-up period among the included studies was three years, and this systematic review failed to assess the treatment effects for these patients in their adulthood, which is one of the main limitations of this systematic review.

Therefore, with regards to the low quality of evidence and limitations in this systematic review, only short-term benefits of orthopaedic appliances for class III malocclusion in children could be suggested.

\section{Practice points}

- Orthopaedic treatments may be effective for class III malocclusion in children in the short term.

Hu Long, Fan Jian and Wenli Lai Department of Orthodontics, State Key Laboratory of Oral Diseases, West China Hospital of Stomatology, Chengdu, China

1. Vaughn GA, Mason B, Moon HB, Turley PK. The effects of maxillary protraction therapy with or without rapid palatal expansion: a prospective, randomized clinical trial. $\mathrm{Am}$ J Orthod Dentofacial Orthop 2005; 128: 299-309.

Evidence-Based Dentistry (2014) 15, 21-22. doi: 10.1038/sj.ebd.6400987 